\title{
Survey on Grid Based Energy Efficient Routing Protocols in Wireless Sensor Networks
}

\author{
Sonal Verma \\ M.Tech CS-II ${ }^{\text {nd }}$ year \\ ABES Engineering College \\ Ghaziabad, India
}

\author{
Ravi Kumar \\ Assistant Professor in \\ CSE Department \\ ABES Engineering College \\ Ghaziabad, India
}

\author{
Prabhat Singh \\ Assistant Professor in \\ CSE Department \\ ABES Engineering College \\ Ghaziabad, India
}

\begin{abstract}
Sensor nodes in Wireless sensor networks (WSN's) have limited energy, range, memory and computational power. In WSN, it is an important task to send measured data at regular intervals from an area of interest for time sensitive applications to a base station or sink for further processing to meet the enduser queries. For reducing energy consumption grid structure was used now-a-days. With the grid structure in place, query and data needs to be propagated to the grid points only. Since the sensor nodes have limited battery power, therefore, approaches have been proposed over the years to reduce energy consumption and to prolong network lifetimes. These approaches in WSN are called as Energy Efficient Routing (EER) protocols. In this paper, a comprehensive list of the grid based EER protocols have been studied with their relative advantages and disadvantages.
\end{abstract}

\section{General Terms}

Grid Based Wireless Sensor Network

\section{Keywords}

Cell, cell head, grid-based, energy efficient routing, source, sink, mobile sink group; data dissemination; wireless sensor networks.

\section{INTRODUCTION}

A wireless sensor network (WSN) consists of a large number of tiny sensor nodes deployed over a geographical area which we also called as sensing field. Each node in a sensing field is a low power device and is equipped with computing, wireless communication and sensing abilities. Wireless sensor network has been applied in different environments, including disaster relief operations, wildlife monitoring, seismic data collection, machine failure diagnosis, structural health monitoring, home security and military intelligence gathering. The sensors predeployed on a variety of environments, established a wireless network infrastructure to communicate and exchange information in a monitor area. Sensor Networks use data-centric approaches for data dissemination from source to sink. This is because sensor nodes are randomly deployed in large number over a sensing, assigning unique identification number to each sensor is not possible.

Although sensors continuously sense and propagate data in an environment, the power supply of each sensor is constrained. It is infeasible to recharge or replace the batteries of the sensors in such a large scale sensor network, yet a WSN is designed to last.
WSN is a class of ad hoc network and its routing techniques widely differ from that of the traditional ad hoc networks. This is due to its energy constrained nature. So rather than focusing on routes with shortest distance, minimum delay or maximum bandwidth, WSN routing approaches are compelled to find out routes that ultimately result in prolonged network life time.

Important challenge to the design of a large wireless sensor network is energy efficient problem. Therefore, for this purpose, considerable research has been conducted on routing data in WSN where the main focus has been on reducing energy consumption giving rise to a new class of routing called Energy Efficient Routing (EER).

In this paper we focus on the dominant Grid based EER protocols with proper classification.

\subsection{Grid-Based Protocols}

The main goal of any Energy Efficient Routing (EER) protocol for WSN is to maximize network lifetime by minimizing energy consumption in end-to-end transmission.

The whole sensing field is divided into a number of square shape cells each of size $\alpha \times \alpha$. These all cells when seeing collectively together forms a grid structure. The main reason for constructing a grid structure is to improve energy consumption by enabling the sensors located at grid points to acquire the forwarding information. From one grid point say $\mathrm{P} 1=(\mathrm{x}, \mathrm{y})$ the location of another grid point say $\mathrm{P} 2=(\mathrm{xi}, \mathrm{yj})$ can be calculated as:

$\{x i=x+i \alpha, y j=y+j \alpha ; i, j= \pm 0, \pm 1, \pm 2, \ldots\}$.

Figure 1 shows the grid structure consisting of a number of cells each of size $\alpha$ with a source and a sink.

A source or sink whosoever is responsible for grid construction calculates the locations of its four neighbouring dissemination points given its location (x, y) and cell size $\alpha$. In particular, a source refers to a sensor node that generates sensing data to report about a stimulus, which is a target or an event of interest. A sink is a user that collects these data reports from the sensor network. Sensor nodes are stationary, sinks can move though. As a target or an event of interest appears in a sensing field, the source generates data reports and the inquirers with mobile devices collect these data reports.

In WSNs, mobility is of great importance for routing and data dissemination purposes (in order to have better connectivity, wider coverage or to support multiple missions) and poses greater challenges. In particular, sink mobility requires energy - 
efficient protocols to guarantee data delivery originated from source sensors toward mobile sinks.

This paper discusses several routing and data dissemination protocols for mobile WSNs by using a grid structure.

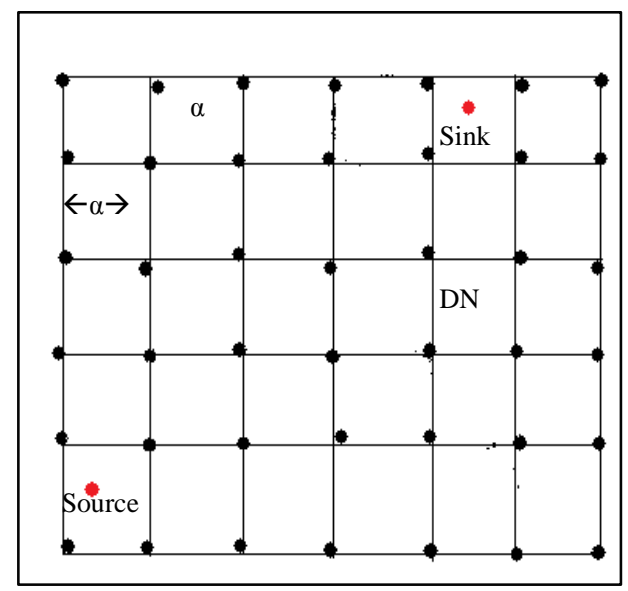

Fig 1: Grid structure

\section{BACKGROUND}

Wireless Sensor Network (WSN) consists of many low cost, low-powered sensor nodes that communicate with each other through wireless medium. For accomplishing this data dissemination task from source node to sink sensor node several protocols have been proposed in recent years.

Gossiping [1] provides an improvement over the straight forward flooding mechanism and also overcomes the implosion problem with flooding. In Gossiping, a packet is not broadcasted to all the neighbours, rather only to a single one chosen randomly which in turn randomly forward the packet to one of its neighbours including the one from which it received the packet. This process continues until the ultimate destination is reached. Although Gossiping reduces energy consumption over flooding to a great extent, but it heavily suffers from long propagation delay.

In Adaptive Protocols for Information Dissemination in Wireless Sensor Networks (SPIN) [2], data dissemination takes place with the help of ADV and REQ messages. A node sends an ADV message to all its neighbours if it wants to send some data, those nodes who are interested in receiving data sends a REQ message in response to the ADV message. The issuer of the ADV message sends the real data to all REQ message issuers via DATA message to complete the whole process. Although, SPIN protocol overcame the limitations of Implosion, Overlapping, and Resource Blindness of the traditional data flooding protocols and also leads to a reduction in energy consumption but it does not guarantee the delivery of data in case the potential neighbours are far away from the ADV message generator (i.e. source node) and the nodes in between the source and destination are not interested in the new data.

GAF [3] reduces energy consumption by turning off unnecessary nodes within transmission range for energy conservation. However, in sensor networks, the detection range of most sensor nodes is smaller than their transmission range. To detect events in a timely fashion, it is necessary to keep a certain sensing coverage.

Directed Diffusion (DD) [4] consists of 3 steps: interest propagation, gradient setup, and path reinforcements. An interest is a message that describes the sensing task about which the sensor network has to acquire data. It reinforced path or paths between sink(s) and source(s) by setting up gradients for data to flow from source to sinks. DD doesn't create any clusters or grid and treat entire sensing field as one contiguous area which leads to higher energy consumption and it also requires frequent location updates from sink (s) so that the direction of sending future data reports could be known which also had a adverse impact as it leads to increased collisions in wireless transmissions.

None of the existing approaches provides a scalable and efficient solution for routing data from source to sink in wireless sensor networks. Also, source and sink mobility brings new challenges to data dissemination in large-scale sensor networks and none of the above protocols addresses this problem. Keeping all the sensor nodes active at all times increases energy consumption of the entire network as well. Therefore, we are discussing about the grid-based protocols that considers the mobility characteristic of the source as well as the sink that try to resolves the above problems.

\section{SURVEY \\ 3.1 TTDD}

TTDD uses a grid based approach for routing data from a source to multiple, mobile sinks. Proactive grid construction is initiated by each source appearing in the sensing field and only the grid points sensors need to acquire the forwarding information.

In TTDD [5] whenever a sink fires a query, it has to traverses two tires to reach a source i.e., the lower and the higher tier. The lower tier form cells of the grid and is within the local grid square of the sink's current location whereas the higher tier is made of the dissemination nodes on the grid.

Whenever a sink needs data it fires a query within a cell so as to discover nearby dissemination nodes. When the query reaches to the immediate dissemination node for the sink, it is forwarded to its upstream dissemination node toward the source, which in turns further forwards the query, until it reaches either the source or a dissemination node that is already receiving data from the source. The Data forwarding is done on the same path but in reverse order as during the query forwarding process, each dissemination node stores the location of the downstream dissemination node from which it received the query. Figure 2 is showing the two tier query and data forwarding process between source A and sink S1 and S2.

This approach improves energy consumption as compared to flooding strategy used earlier by making a grid structure and choosing grid points sensor to be involved in query and data dissemination process.

TTDD allows query and data to be forwarded either horizontally or vertically because of which a query or data has to cover a larger area and minimum distance can't be used, i.e., no diagonal-forwarding is there.

In TTDD, whenever a sink from its original position moves a distance that is greater than a cell size away then local flooding of query takes place and a new DN is selected which does query forwarding until it finds a DN that is already receiving data from the source. This local flooding of query seems to be an inefficient strategy as flooding to all nearby nodes leads to higher energy consumption and introduce delays. A better strategy that can be adopted is to use the path extension method. 
Also it includes the overhead of selecting Immediate and Primary agents for handling sink mobility.

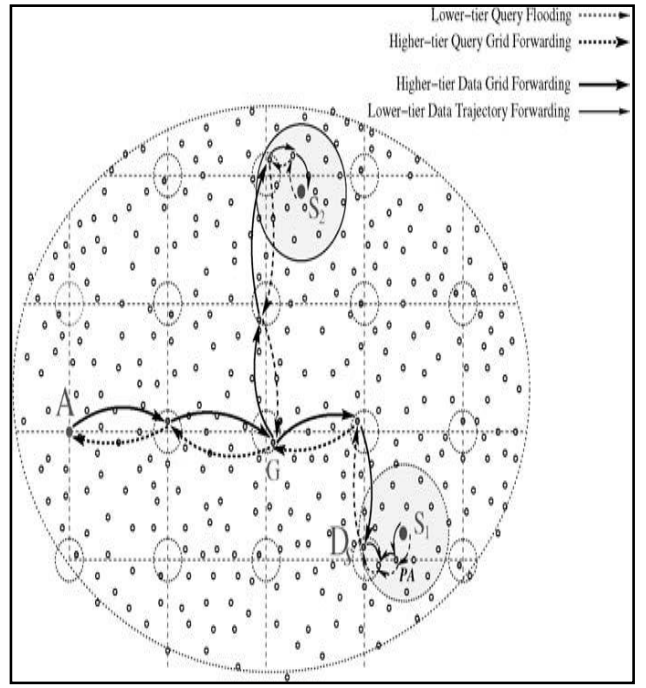

Figure 2: Two-tier query and data forwarding[5].

Another drawback of TTDD is that a sink can't move faster than the local forwarding states being updated. TTDD works well only for event-driven system and is not suitable when source moves fast or appear and disappear frequently.

TTDD's DN failure handling strategy is not very effective as it introduces delays because it is an on-demand failure handling strategy and selection of a new DN is done the similar way as was done during grid construction and no pre-information was gathered for electing a new DN and for detecting failures except time-out strategy while query or data forwarding.

Another major disadvantage of TTDD is that it constructs grid on per-source-basis which costs major overhead if the number of sources are more and also consumes a significant amount of energy.

Moreover, the maintenance of source based grid structure is very difficult because with the mobility of event, its source nodes also changes.

\subsection{EEDD}

In Energy-Efficient Data-Dissemination protocol, EEDD [6], virtual-grid-based two-level architecture is adopted to extend the lifetime of a sensor network. The 2 levels of the grid are: Coarse and Fine level. At Coarse level, only some necessary nodes are kept active only via a detection process and rest nodes goes to long term sleep. At fine level, each grid is divided into several sub-grids and working nodes in each sub-grid will alternatively stay active from short term sleep according to a schedule. Data dissemination strategies are characterized into three types, i.e. Target location aware, Target area aware and Target location unaware. In EEDD, query and data is forwarded using diagonal-first routing strategy but query and data dissemination path are normally different from each other. In target location aware strategy, as the target's location is known, query can be forwarded using the diagonal-first routing strategy until the query reaches the grid head whose grid ID has the same vertical or horizon coordinate value as that of the source grid. In Target area aware strategy, query is flooded to all grid heads in the sub-area where the source resides. In Target location unaware strategy, query flooding is done throughout the field and it reaches all grid heads in the field and the nodes which have event in their sensing field sends the relevant data back to the sink. Figure 3 is showing that sub grid $\mathrm{ABCD}$ is divided into four sub-subgrids P1, P2, P3 and P4 and the diagonal first routing strategy is used for target location aware data dissemination from multiple sources to a single sink.

EEDD's major advantage is that it does diagonal forwarding for query and data dissemination purposes. It uses the technique of entry checking in its forwarding table at each node for detecting the formation of forwarding loop which forms when a sink node moves from one grid to other(s) and finally reaches to the original one shortly and removes it by deleting the old forwarding entry. For handling source mobility, EEDD introduces the concept of intelligence by designating the sensor nodes as Normal or Smart. A Normal node aggregates information about an event on-demand whereas a Smart sensor nodes proactively collects information about any event as soon as it appears in its sensing field.

This approach turns active nodes into sleep modes which on one hand absolutely saves energy but also introduces additional delays, on the other hand.

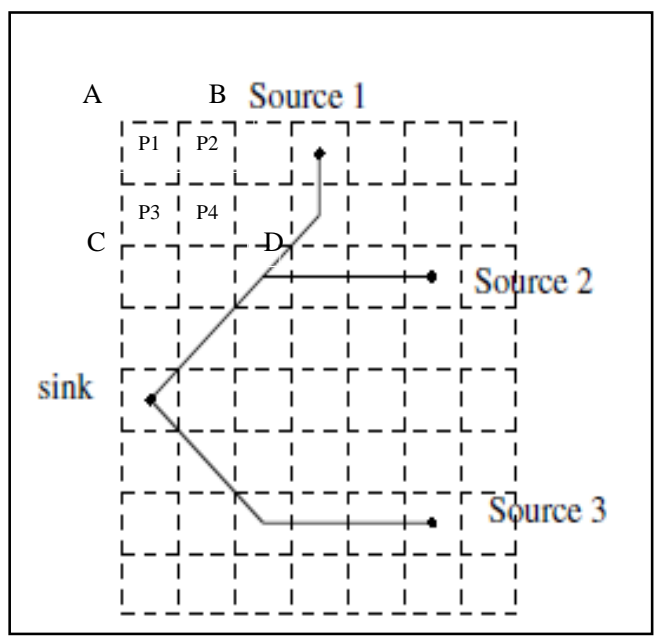

Figure 3: Diagonal first routing strategy in EEDD[6].

If the sink moves faster than the local forwarding states being updated then path extension becomes longer resulting inefficient energy consumption and longer delays. So, some sort of constraints should be employed.

Its another drawback is that EEDD doesn't maintain query routing path to send data back to sink later, instead, it searches the path to route data to the sink which, undoubtedly reduces collisions but leads to more energy consumption and also introduces delay.

Moreover, EEDD grid head maintenance strategy is action-ondemand which introduces delays during query or data forwarding process on failure of one or more DN

\subsection{GBDD}

In Grid Based Data Dissemination protocol, GBDD [7] the grid is constructed by the first sink when no valid grid is present and it uses dual radio mode of a sensor node for grid construction. All nodes within a cell form a cluster electing one of nodes at corners of the cell as cluster head. All nodes communicate with each other using high power radio $(\mathrm{RH})$ whereas cluster head DNs communicates with nodes in its ADZ using low power radio (RL). When sink sends query message then sensor nodes which is in the event's sensing range sends path set up message Msetup to their cluster head DN called Source Dissemination 
Node (SDN). SDN forwards this message to its upstream DN which further forwards the query and so on until it reaches at sink or a common dissemination node between multiple sinks. During grid formation process all DNs gather information about upstream neighbour which is used for setting up the initial path for query and data flow. Figure 4 is showing how the query and data is forwarded from SDN A to Sink S1.

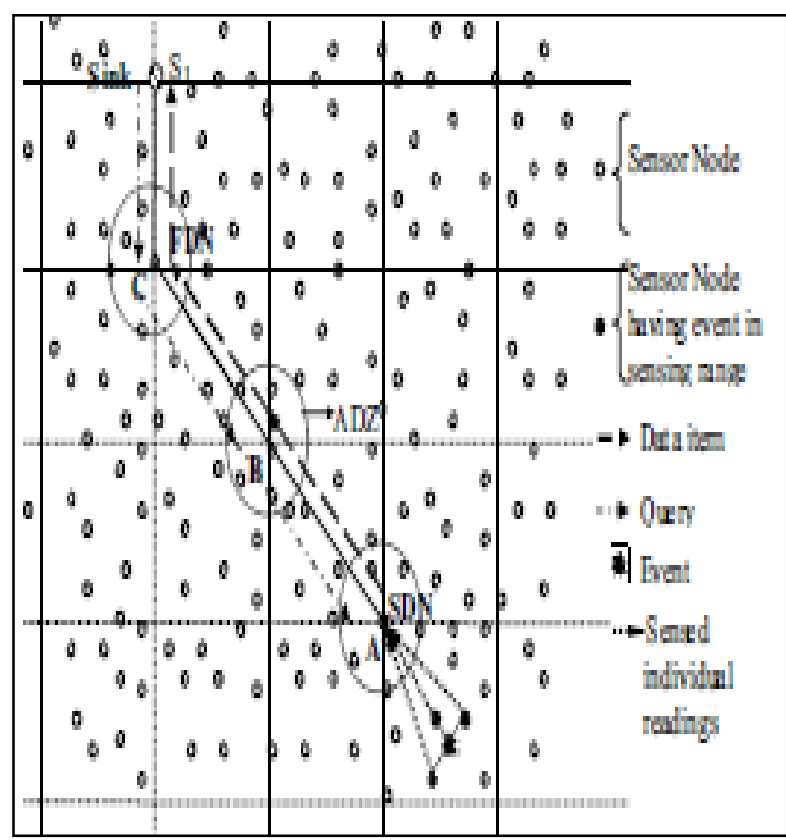

Figure 4: Setting Query and Data flow path[7].

GBDD constructs grid only when sink first time appears in the sensing field hence saving energy consumption which may otherwise couldn't be possible if it constructs grid with the appearance of every source as in case of TTDD. Its other advantage is that any node can communicate with any other node if it is within its high radio transmission range so diagonal forwarding can also be done.

However, in GBDD while forming ADZ by a DN, all nodes has to send a tuple containing coordinates and residue energy to every other node which not only occupies storage memory of the sensor but energy consumption also increases significantly. The solution is that this tuple value may be sent to some nodes but not to all for energy saving purposes.

Also, grid exists only during that life period which is given by the first sink during the grid formation process and it will be used only by those sinks which appears during the given time period and once the timer expires any current query forwarding or data sharing information might also get lost.

GBDD during handling event mobility assumes that if event moves to a cell where none of its corner node is either old source dissemination node or a dissemination node on existing path then the corner node of this new cell broadcast probe message to their respective one hop neighbour DNs only and one of them will surely finds a DN on existing path or find its old SDN so source chooses that node as new SDN and path is completed. But this is not practically always possible and event may move to a cell where none if its corner node DNs finds a node that has either old SDN or DN on existing path then in that case this approach doesn't gives any solution.

\subsection{Data Dissemination Scheme for Mobile Sink Group Based on movement behaviour}

This introduces a mobile sink group based data dissemination scheme [8] which considers both the macro and micro mobility of the mobile sink group while acknowledging the continuous movement of the group. It tries to overcome the limitations and problems occurred by previous scheme such as in geocasting [9] scheme that could not trace the macro movement of the group, RBDD [10] which can't overcome its unreachability problem, i.e., some mobile sinks could not receive the source data. This scheme creates a virtual grid infrastructure, initially and a grid ID is assigned to every sub grid in the virtual grid. After that a leader sink is chosen which find four points, i.e., uppermost, undermost, leftmost, and rightmost on the circle of the group, and compare their grid-Ids to know which grid (or grids) the group is located in. This process is repeated periodically and four vertices of the tetragon which fully includes the mobile sink group are known as grid-points whose information is being sent to the source node which send its data to the boundary lines of the four grid-points. Source uses the geographic routing [11] to send the sensed data and grid-points toward the closest grid-point from itself. The sensed data is saved for a certain time period by all the sensor nodes on the boundary lines of the tetragon. The sink group will receive the source's data when it moves out from its local grid. Figure 5 shows an example of the data dissemination mechanism from Source to a sink group whose reference coordinates are $(\mathrm{ABDC})$ at time $\mathrm{t} 1$ and (JHKE) at time t4. To handle the situation when a group has only micro mobility, a timer is chosen which is proportional to the tetragon size. If the leader sink grid-points are not changed until the timer is over, then the source's data is requested by the leader sink.

This method works well to disseminate data to mobile sink group. But it has negative points too.

It may be possible that all sinks in a mobile sink group doesn't want data about the same event. In that case the boundary sensor nodes of the outer tetragon will be flooded with the information from multiple events of interest. As we know that the storage capacity of every sensor nodes is limited, hence, it leads to information loss due to buffer overflow condition and it also leads to excessive energy consumption. In other words, we can say that this scheme is inefficient for handling multiple sources.

To handle the only micro mobility condition (i.e., when mobile sinks randomly move in its group limited to some geographical area) geographically restricted flooding is used if there is not any macro mobility until some timer expires to receive sensed data. It introduces delays because each of the sensor nodes in a sink has to wait till the timer expires and if the closest grid point doesn't contain the requested data then the timer value is extended further resulted more time consumption. So some trade-off regarding the timer value (very small to very large related to energy consumption) must be dealt with. 


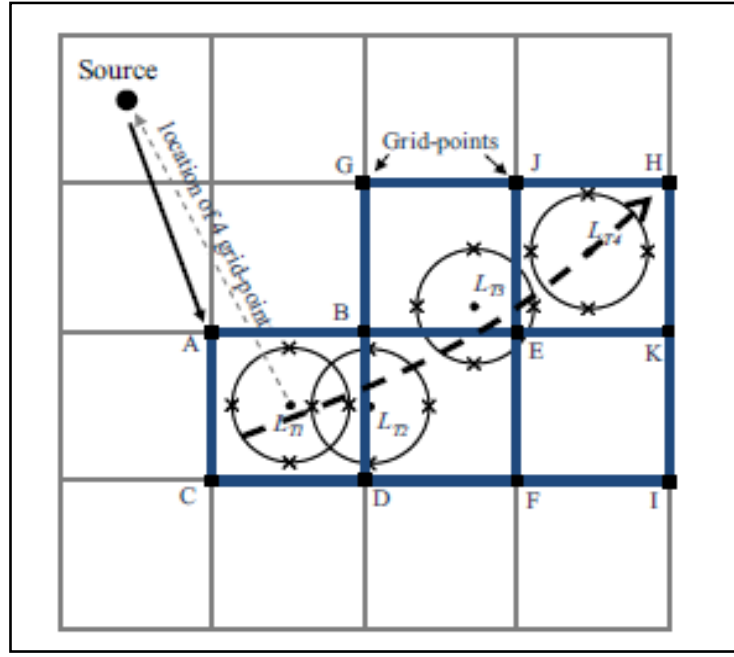

Fig. 5: Data dissemination from source to sink group[8].

\subsection{EEGDG}

Energy efficient grid-chain based data gathering (EEGDG) [10] merges grid and clustering technique together for data forwarding purposes. In this approach, whole sensing field is divided into a number of cells and by clustering technique each cell chooses a grid node (GN) that connect each cluster and transmits the data to the base station as is shown in figure 6 . For avoiding uneven energy consumption, each GN is randomly chosen by turns and all nodes in a sub-area transmit their data to the specified GN. Finally, all the GN is connected together forming a chain.

Though EEGDG helps to improve the network lifetime, it suffers from the disadvantage that if the chain distance between the nodes is long than the geographic straightforward distance then the consumed energy is higher than that in the latter case. Another negative point is that it doesn't take into consideration the mobility of nodes.

\subsection{CBDAS}

Cycle-Based Data Aggregation Scheme (CBDAS) for gridbased wireless sensor networks (WSNs) constructs a cyclic chain for transmitting the data to the base station (BS). It is an improvement over various chain-based protocols developed previously, for example, the PEGASIS [13] protocol which forms a cyclic chain by connecting all sensor nodes with a greedy algorithm. Data is transmitted from one chain end to the other where the BS is located.

CBDAS [14] works by dividing the whole sensor field into two dimensional logical grid of MXN cells, where $\mathrm{N}$ is even. In this every node keep a store of its own sequence number, geographic location, cell ID, and the current cell head.

For the first round of data transmission, the node with the smallest sequence number acts as the cell head in each cell. But for the second turn, cell head for each cell is the one which has the most residual energy in comparison to other sensor nodes in

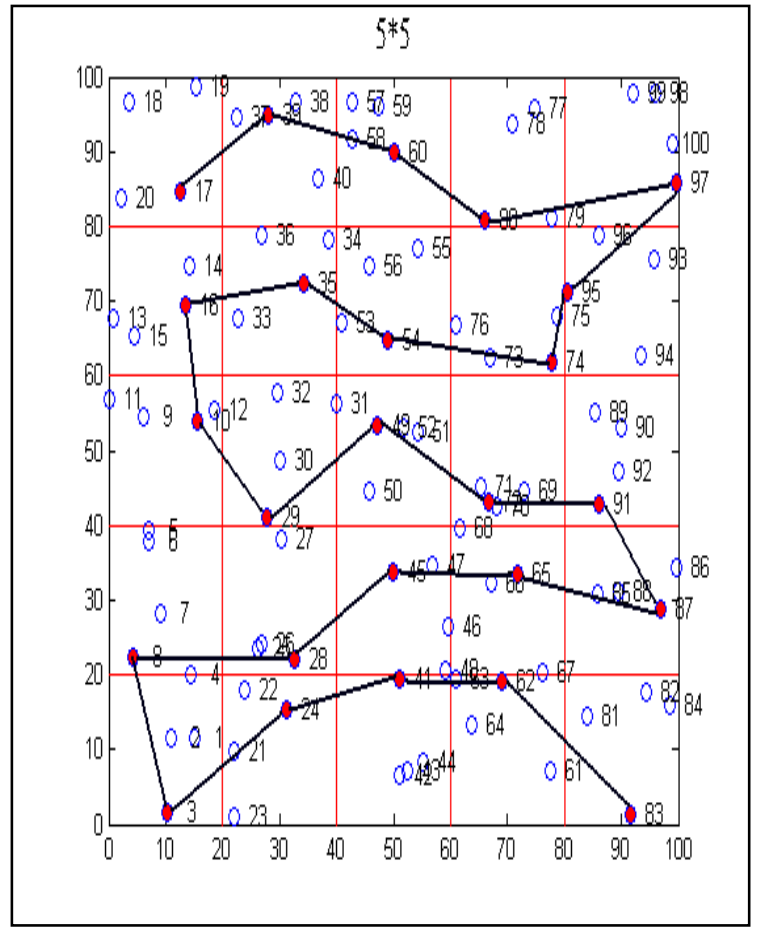

Fig. 6: Date transmission in EEGDG with number of grids $5 \times 5[10]$.

the same cell. After that each cell head starts finding its downlink cell head in its neighboring cell in clockwise direction and make use of the Cycle_forming packet and Cycle_reply packet, respectively to request the formation of a cyclic chain. Finally, each cell's Cell head are linked together forming a cyclic chain throughout the sensor network. A cycle leader is chosen by the BS which is nearest to it, for the case of first data transmission. To receive data from entire sensor network, BS requests its cycle leader which in turn sends two tokens t1 (in clockwise direction) and $\mathrm{t} 2$ (in anticlockwise direction) to its two neighbouring cell heads, respectively along the cyclic chain. The cycle will be disconnected at the cell head (say e1 and e2) at which the two tokens meet resulting the formation of two separate ends through which the aggregated data from previous cell head's will be transmitted to the cycle leader in the opposite direction and then to the BS as shown in figure 7 .

No doubt, CBDAS extends the lifetime of sensor nodes so as to prolong the lifetime of the whole network via its chain formation process in the grid structure.

The problem with this protocol is that it can't efficiently manages the mobility of nodes as it assumes in its network model that all sensor nodes are static once deployed, which can't be always possible. Consider the case in which the sensor nodes are mobile and one or more cell head's of the cyclic chain moves from its initial position during the data sending process. Then in that case path extension could be done but up to what limit it can be done, so we can choose another cell head (may be on-demand strategy will be adopted) which leads to additional delays and degraded performance of the whole scheme.

Also, the tokens that a cycle leader sent for data aggregation purposes also serves as major points of failure. Any error or deviation or loss of any one or both of them affects majorly the 
network's performance. This scheme doesn't mention any proper way for dealing with this sort of problem.

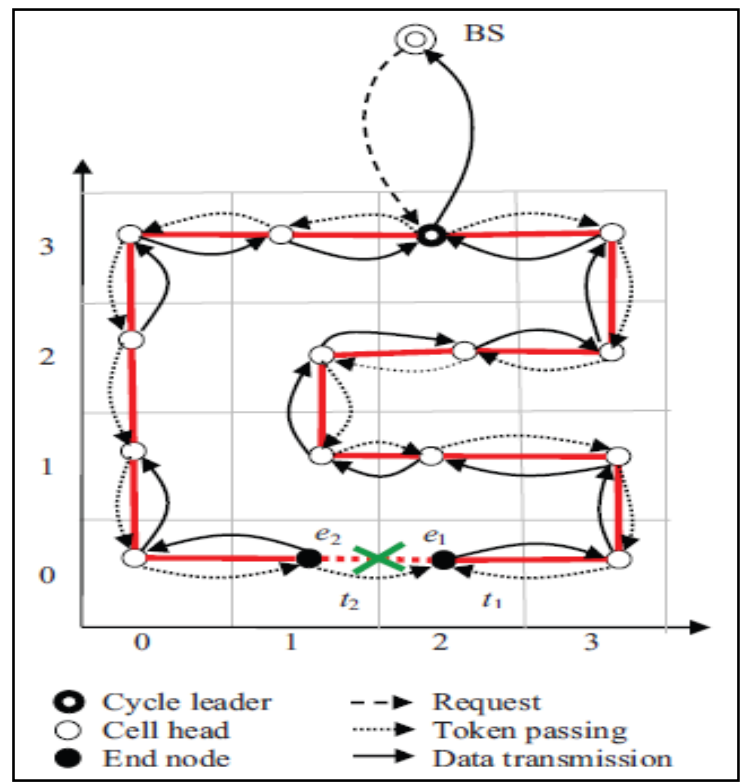

Fig 7: Data gathering for each round[14].

This token strategy leads to two paths for sending the aggregated data which causes delays and major energy consumption as the aggregated data consumes greater energy. A better strategy is to adopt the hierarchal scheme which can be employed by sending tokens equal to the number of columns in the virtual grid structure.

\section{CONCLUSION}

In this paper, a comprehensive list of the various grid based EER protocols for WSN has been studied. Through this study, we came to know that there are plenty of issues which these protocols are failed to address. One doesn't consider the nodes mobility but other one did but can't maintain the network efficiency effectively. There are other issues also like proper frequency utilization, cost effective localization etc. So, it is expected that upcoming protocols would consider these shortcomings and develop highly energy efficient grid based routing protocols.

\section{REFERENCES}

[1] S. M. Hedetniemi, S. T. Hedetniemi, and A L. Liestman, "A survey of gossiping and broadcasting in communication networks", Networks, 18(4): pp. 319-349, 1988.

[2] W. Heinzelman, 1. Kulik, and H. Balakrishnan, "Adaptive protocols for information dissemination in wireless sensor networks", in Proceedings of the 5th Annual ACM/IEEE International Conference on Mobile Computing and Networking (MobiCom_99), Seattle, WA, Aug. 1999.

[3] Y. Xu, J. Heidemann, and D. Estrin, "Geography-informed energy conservation for ad hoc networks" in Proceedings of the Seventh ACM/IEEE International Conference on Mobile Computing and Networking (MOBICOM), July 2001.

[4] c. Intanagonwiwat, R. Govindan, and D. Estrin, "Directed diffusion: a scalable and robust communication paradigm for sensor networks " in Proceedings of the 6th Annual ACMIIEEE International Conference on Mobile Computing and Networking (MobiCom_OO), Boston, MA, Aug. 2000.

[5] Haiyun Luo, Fan Ye, Jerry Cheng, Songwu Lu and Lixia Zhang," TTDD: Two-Tier Data Dissemination In LargeScale Wireless Sensor Networks In Wireless Networks", Pages 161-175, 2005.

[6] Zehua Zhou, Xiaojing Xiang, Xin Wang and Jianping Pan, "An Energy-Efficient Data-Dissemination Protocol in Wireless Sensor Networks" in Proceedings of the 2006 International Symposium on a World of Wireless, Mobile and Multimedia Networks (WoWMoM'06) 0-7695-25938/06, 2006 IEEE.

[7] T.P. Sharma, R.C. Joshi, Manoj Misra, "GBDD: Grid Based Data Dissemination in Wireless Sensor Networks", 2008 IEEE.

[8] Jeongcheol Lee, Hosung Park, Seungmin Oh, Yongbin Yim, and Sang-Ha Kim, "Data Dissemination Scheme for Mobile Sink Group based on Movement Behaviour in Wireless Sensor Networks" in 2011 International Conference on Advanced Information Networking and Applications, 1550-445X/11, IEEE.

[9] I. Stojmenovic, "Geocasting with guaranteed delivery in sensor networks," IEEE Wireless Communications, Vol. 11, No. 6, pp. 29-37, Dec. 2004.

[10] H. Lee, J. Lee, S. Oh, and S. Kim, "Data Dissemination Scheme for Wireless Sensor Network with Mobile Sink Groups," IEEE International Symposium on PIMRC, Sep. 2010.

[11] B. Karp, H.T. Kung, "GPSR: greedy perimeter stateless routing for wireless networks," in Proc. 6th Annual Intl. Conf. Mobile Computing and Networking, pp. 243-254, 2000.

[12] Yung-Fa Huang, Li-Chu Yang and Jen-Yung Lin, "An Efficient Energy Data Gathering Based on Grid-Chain for Wireless Sensor Networks", 2012, IEEE.

[13] S. Lindsey and C. S. Raghavendra, "PEGASIS: PowerEfficient Gathering in Sensor Information Systems," Proceedings of the IEEE Aerospace Conference, Vol. 3, pp 1125-1130, March 2002.

[14] Yung-Kuei Chiang, Neng-Chung Wang, and Chih-Hung Hsieh, " Cycle-Based Data Aggregation for Grid-Based Wireless Sensor Networks" in 2013 Seventh International Conference on Innovative Mobile and Internet Services in Ubiquitous Computing, 978-0-7695-4974-3/13 ， 2013 IEEE. 